\title{
From Gregor Mendel to Eric Davidson: Mathematical Models and Basic Principles in Biology
}

\author{
UTE DEICHMANN
}

\begin{abstract}
Mathematical models have been widespread in biology since its emergence as a modern experimental science in the 19th century. Focusing on models in developmental biology and heredity, this article (1) presents the properties and epistemological basis of pertinent mathematical models in biology from Mendel's model of heredity in the 19th century to Eric Davidson's model of developmental gene regulatory networks in the 21st; (2) shows that the models differ not only in their epistemologies but also in regard to explicitly or implicitly taking into account basic biological principles, in particular those of biological specificity (that became, in part, replaced by genetic information) and genetic causality. The article claims that models disregarding these principles did not impact the direction of biological research in a lasting way, although some of them, such as D'Arcy Thompson's models of biological form, were widely read and admired and others, such as Turing's models of development, stimulated research in other fields. Moreover, it suggests that successful models were not purely mathematical descriptions or simulations of biological phenomena but were based on inductive, as well as hypotheticodeductive, methodology. The recent availability of large amounts of sequencing data and new computational methodology tremendously facilitates system approaches and pattern recognition in many fields of research. Although these new technologies have given rise to claims that correlation is replacing experimentation and causal analysis, the article argues that the inductive and hypothetico-deductive experimental methodologies have remained fundamentally important as long as causal-mechanistic explanations of complex systems are pursued.
\end{abstract}

Keywords: gene regulatory networks, Eric Davidson, Alan Turing, D’Arcy Thompson, genetic causality, biological specificity.

\section{INTRODUCTION}

Thee statements on mathematical modeling in biology are pertinent for this article:

"The model provides a means for experimentally testing the relevance and consistency of a [causal-mechanistic] concept." (Peter and Davidson, 2015)

"The denouement of intelligibility began with Galileo and Newton, when knowledge of the physical world was freed from the requirement of a causal description, the Aristotelian ground of

Jacques Loeb Centre for the History and Philosophy of the Life Sciences, Ben-Gurion University of the Negev, Beersheba, Israel.

(C) Ute Deichmann, 2019. Published by Mary Ann Liebert, Inc. This Open Access article is distributed under the terms of the Creative Commons Attribution Noncommercial License (http://creativecommons.org/licenses/by-nc/4.0/) which permits any noncommercial use, distribution, and reproduction in any medium, provided the original author(s) and the source are credited. 
science, and became associated with mathematical equations serving as quantitative descriptions of the effects exerted by hypothesized relationships." (Dougherty and Bittner, 2011)

"There is now a better way [than modeling]. Petabytes allow us to say: 'Correlation is enough.' We can stop looking for models. We can analyze the data without hypotheses about what it might show." (Anderson, 2008)

These statements illustrate three contrasting views of mathematical modeling in the current research on the biology of complex systems such as the cell or development. While experimental molecular system biologists such as Eric Davidson view models as predictive hypotheses of reality, engineers and computational biologists such as Dougherty and Bittner conceive models as mathematical descriptions. In some areas of current fast-rising, big data-based biomedical research, models, as well as hypotheses, are marginalized or replaced with data-driven correlations.

This article neither attempts to give a comprehensive overview of modeling in developmental biology and heredity nor to suggest a definition of "model." It uses the term in a broad sense, distinguishing three kinds of quantitative models in biology: (1) Models as descriptions of observations or experimental data that are used to represent findings in mathematical language. (2) Models as hypotheses based on observations or experimental data. They are used to test the assumptions resulting from experimentation, make predictions, and provide conceptual frames for further experimenting. (3) Models as simulations of observations or experimental data that are not based on data. They can be adjusted to fit data, but they do not explain or describe them mathematically and usually are not predictive.

Unlike mathematical models in physics that often are derived from assumptions based on fundamental laws or axioms (Gunawardena, 2014), quantitative models in biology have strongly differed regarding function and explanatory power among researchers in various fields.

Most biologists have used models as tools for representation, simplification, explanation, or handling data, but some have perceived mathematics as an expression of biological reality, most prominently D'Arcy Thompson and Mendel (see Deichmann, 2010). Despite the fact that the history of biology reveals a widespread skeptical attitude toward the use of mathematics (Brenner, 1999; Fox Keller, 2002), some of the quantitative biological models have been of far-reaching importance (Brenner, 1999; Fox Keller, 2002; Gunawardena, 2012, 2013, 2014).

In this article I focus on quantitative modeling in research that aims at understanding fundamental features of development and heredity. I do not include a discussion of the comparatively widespread models in physiology, ecology, population genetics or origin of life, and artificial life research, many of which, especially in the latter fields, are computational simulations.

This article, first, presents pertinent models and their reception in the 19th and 20th centuries from Mendel to the modeling of the genetic code and examine their contrasting epistemologies as indicated above. Unlike the illuminating work by Fox Keller (2002), the article does not aim at revealing "explanatory styles" and the "diversity of epistemological goals" (Fox Keller, 2002, p. 300). Rather, it tries to understand the fruitfulness of different models by relating them to the differences in the underlying epistemology and to models' regard-or disregard—for basic principles of biology, in particular biological specificity or biological information and genetic or genomic causality (see Deichmann, 2017), the importance of which has also been indicated by Brenner (1999): "biological systems are informationprocessing machines, and this must be an essential part of any theory we may construct."

The article, second, analyzes mathematical modeling in research on complex systems in the 21 st century. It focuses on models of developmental gene regulatory networks (GRNs), in particular those by Eric Davidson who combined the use of large amounts of DNA sequencing and gene expression data with painstaking perturbation experiments and causal modeling. This approach, in which the principles of genetic information and genomic causality were central, and its explanatory scope are then compared with those of "data-only" approaches in biomedical research that marginalize or abandon causal analyses and modeling.

\section{NINETEENTH TO MID-20TH CENTURY MATHEMATICAL MODELS}

\subsection{Models as hypotheses}

2.1.1. Mendel's model of the formation of plant hybrids. The most prominent example of a model that has remained crucially important until the present day is Mendel's "law governing the formation and development of hybrids" (Mendel, 1866, p. 1), which was based on years of his research on hybridization 
in Pisum. Mendel reduced the complex features of hybridization to the behavior of hypothetical elements of heredity in a quantitative way that resulted in his suggesting a quasi-binomial formula for the combination of the dominant and recessive "elements" "A" and "a" during fertilization: $A / A+A / a+a / A+a / a=A$ $+2 \mathrm{Aa}+\mathrm{a}$ [for details and an explanation of the mistake in the equation, see Deichmann (2010) and references therein].

Mendel was not the first biologist to quantify his results. Quantification and correlation were important practices, for example, in 19th-century biogeography and plant morphology (Gliboff, 1999; Teicher, 2014; Droescher, 2015). Using empirical data and a statistical approach based on measuring cell features, Franz Unger, a pioneer of cell theory, and Carl Naegeli, a prominent botanist, formulated various laws of growth in plants. Naegeli aimed at demonstrating "the existence of an architectural plan" in cell distribution in growing plants, concluding that "it is possible to find terms of absolute (mathematical) form for physiology and systematics" (Droescher, 2015). Unger's and Naegeli's models consisted largely of numerical correlations. Naegeli's model was based on enormous amounts of poorly-defined empirical data and equations (Droescher, 2015). Unger's and Naegeli's models did not influence subsequent botanical research by others. Moreover, Naegeli, a renowned botanist at the University of Munich, was entrenched in his paradigm of the phenomenology of morphological developmental form to such an extent that he misapprehended and misjudged Mendel's approach when the latter submitted his findings to Naegeli. Mendel's hope for fruitful scientific exchange with a colleague did not fulfil (Mendel, 1866-1873).

For Mendel, mathematics was of fundamental importance for understanding life processes. For example, he was the first to establish statistical relationships between the number of forms in separate generations because he considered this to be "the only correct way" to solve a question that had a far-reaching importance for the "development of organic forms" (Mendel, 1866, translation UD).

In contrast to other quantitative models of his time, Mendel's model

- used large amounts of quantitative experimental data in the creation of a simplified model, with the help of which he was able to predict experimental results probabilistically. Unlike other botanists at the time who carried out hybridization experiments, Mendel focused his experiments on single characters and not on organisms or species. He introduced the new concept of character pair as a unit of crossing experiments and included only few clearly distinguishable parameters.

- was not descriptive but causal and explanatory. The model was the result of a reasoned generalization of observations followed by the generation of hypotheses, predictions, and experimental hypothesis testing. Mendel causally explained cellular development by the behavior of hypothesized elements in the cell. Thus apart from the new methodology that Mendel created, the most preeminent feature of his model was its prediction of invisible discrete entities, which later gave rise to the concept of "gene." Mendel's model provided the conceptual framework for designing new experiments.

The gene concept was introduced by the Danish botanist Wilhelm Johannsen in 1909 as "for the time being only something like a unit of calculation." It became one of the most powerful abstractions in biology and one of great utility (Lewis, 1995). Although there were assumptions about genes being material entities early on, research in this direction only began in the 1930s, with DNA becoming a major candidate from 1944, that is, ca. 80 years after Mendel's discovery. Therefore, Mendel's model was not a description but as Gunawardena (2012) deduced from the example of the kinetic model of Michaelis and Menten: "Models are not descriptions of reality; they are descriptions of our assumptions about reality." This means that they are mathematical formulations of hypotheses.

Thus the special features that distinguished Mendel's model from other mathematical approaches in the 19th century were simplicity, exactitude, probabilistic prediction, and the proposition of hypothetical entities.

2.1.2. Mathematical modeling of the genetic code. The deciphering of the genetic code during the years after the elucidation of the double helix structure of DNA in 1953 was pursued through mathematical, as well as biochemical, approaches. The complicated path leading to this deciphering has been traced, for example, by Morange (1998, pp. 120-138) and Kay (2000). Physicists here played important roles. Already in 1944 theoretical physicist Erwin Schrödinger speculated on how genetic information might be stored linearly in the material of genes (which he mistakenly believed to be proteins). Schrödinger created the idea of an aperiodic crystal as a model for the central importance of (linearly stored) specific information for individual development and functioning, which was by then believed to be stored in genes, and he was one 
of the first to use the term "code" in regard to genes. Although Schrödinger's model was flawed in several respects, it generated a new vision of biology that would soon impact the new science of molecular biology (Morange, 1998, p. 74). According to Morange (1998, p. 276), Schrödinger's view reflects the mechanistic and deterministic view of Descartes regarding genes and chromosomes.

In their first proposition of the hypothesis of the double helix structure of DNA, Watson and Crick used the word "code" and made it clear that the idea of genetic specificity or "genetic information" would have to be based on a linear "code," not a three dimensional structure, if DNA was proven to be the material of genes: "The phosphate-sugar backbone of our model is completely regular, but any sequence of the pairs of bases can fit into the structure. It follows that in a long molecule many different permutations are possible and it therefore seems likely that the precise sequence of the bases is the code which carries the genetic information" (Watson and Crick, 1953). However, when astrophysicist George Gamow, inspired by this publication, proposed his first theoretical model of a genetic code-an overlapping triplet code (Gamow, 1954) - Watson and Crick were taken by surprise: they had not imagined that the code could in fact be deciphered (Morange, 2001, p. 69). To arrive at his code, Gamow simplified and quantified the complex question of what the DNA sequences should code for. In 1954 he suggested that hereditary properties are characterized by a long number written in a four-digit system, while proteins could be considered as long "words" based on a 20-letter alphabet. The question about the code was thus simplified to the question about the way in which four-digit numbers could be translated into such "words" (Gamow, 1954).

At the time, there was only little knowledge about the determination of protein structure. In the 1930s Linus Pauling demonstrated that proteins' three-dimensional structure was based on weak bonds between amino acid residues (Mirsky and Pauling, 1936). In 1948, following his mistaken claim that antibodies' shapes were induced by antigens and had nothing to do with their amino acid sequence (Pauling, 1940; Pauling and Campbell, 1942a, 1942b), he proposed that the electrophoretic differences between normal and sickle cell hemoglobin were not due to a change in amino acid sequence but only to a change in the folding. Thus, until 1956 the general assumption was that biological specificity of proteins lay in their threedimensional structure, not in their amino acid sequence. This would mean that protein folding, not sequence, should be influenced by the information in the gene.

But Crick, a physicist himself, appreciated Gamow's idea of a code based on the correspondence between sequences of nucleotides and amino acids and removed some of Gamow's errors such as choosing the wrong sample of 20 amino acids. To critically examine Pauling's assumption that the amino acid sequence was irrelevant for protein structure, he convinced his colleague Vernon Ingram to investigate whether the two kinds of hemoglobin also differed in their amino acid sequence, something that Ingram showed conclusively (Crick, 1958). In part, based on this evidence, Crick in 1958 formulated the "sequence hypothesis," according to which the specificity of a piece of nucleic acid is expressed solely by the sequence of its bases and that "this sequence is a (simple) code for the amino acid sequence of a particular protein" (Crick, 1958).

The subsequent efforts to decipher the code transformed a biochemical problem-the relationship between DNA structure and protein synthesis-into an abstract mathematical problem related to numbers. [Kay (2000) pointed to the problem connected with the term "code," as the code is not a code in the technical sense.] Renowned physicists participated in the "code-breaking" efforts, among them, apart from Gamow and Crick, Feynman and Teller. Many different codes were theoretically proposed, overlapping, nonoverlapping, or comma-free codes, as well as solutions to reading the frameshift. It was assumed for some time that physics and information theory were sufficient to work out the genetic code (Kay, 2000). However, experiments refuted many of the hypotheses.

When the nature of the code was finally deciphered biochemically in 1961 by Lothar Matthaei and Marshall W. Nirenberg (Kay, 2000), it was recognized that these theoretical approaches had led to a dead end: The magic number of 20 does not play a role; nature ignores all the mathematical ingenuity involved to solve the frameshift problem-except for signals that mark where the ribosomes are supposed to start, there is nothing in the code itself that enforces the correct reading frame; and the code is redundant (degenerate) (see also Hayes, 1998; Kay, 2000).

However, despite the failure to solve the code, theory and mathematical modeling had been crucially important for finding the solution of the genetic code. A physicist, Gamow, suggested the initial simplification and abstraction that was necessary for building experiments. The notion of information that was furthered and included in biological reasoning by Schrödinger among others, as well as initial reflections on the nature of the code, provided the theoretical groundwork for devising hypotheses and experiments that 
finally led to the elucidation of the nature of the code and the composition of the codons. In hindsight, Carl Woese, a biologist who experienced the fascination at that time with the intellectual elegance and the appeal of the numerology of some of the theoretical codes and the subsequent biochemical denouement, emphasized the importance of this theoretical work. In his opinion, the second period of the code breaking effort, that is, the elucidation of the particular triplet codes for every amino acid, could advance so spectacularly fast, precisely because of the conceptual framework that had already been laid in the theoretical work beforehand (Kay, 2000). It is exactly the interplay of theory and experiment that led first to the notion of genetic code and then to its fast solution.

\subsection{Models as descriptions and simulations}

2.2.1. D'Arcy Thompson's mathematical modeling of organisms' growth and form. In his widely read book On Growth and Form, first published in 1917, British zoologist Wentworth d'Arcy Thompson combined a morphological approach with simple mathematics and a deep knowledge of Greek philosophy to find unifying principles in life's forms. Following Kant, he held that "the criterion of true science lay in its relation to mathematics" (Thompson, [1917] 1942, p. 1). According to Thompson, organic form was predetermined by the physical organization of the system in which it developed, obeying the law of physics in which form was a diagram of forces. His "theory of transformations" aimed at showing how the differences between forms of related species, in particular fish, could be represented geometrically so that one form could be transformed into another one with the help of a simple equation.

Thompson's aim was "to correlate with mathematical statement and physical law certain of the simpler outward phenomena of organic growth and structure or form, while all the while regarding the fabric of the organism, ex hypothesis as a material and mechanical configuration" (Thompson, [1917] 1942, p. 14). As an antimaterialist, he rejected theories that attributed specific properties to particles of the protoplasm, such as chromosomes. In his opinion such an attribution would mean committing the "error of attributing to matter what is due to energy and is manifested in force: or more strictly speaking, of attributing to material particles individually what is due to the energy of their collocation." To him, August Weismann's term of a "hereditary substance" could only mean "that that particular portion of matter is the essential vehicle of a particular charge or distribution of energy, in which is involved the capability of producing motion, or of doing work" (Thompson, [1917] 1942, p. 288). Thompson did not mention Boveri's and Sutton's chromosome theory which they proposed independently in 1903 and 1902, which suggested a causal role of chromosomes for heredity and development, and which, a few years later, was amply confirmed and refined by Thomas H. Morgan and his school.

Thompson based his discussion of form and symmetry on a large amount of biological phenomena. He places considerable emphasis on phenomena described by physical chemist Stéphane Leduc, who claimed to have created artificial life by simulating phenomena such as karyokinesis and organisms' forms with the help of osmotic growth processes (Thompson, [1917] 1942, pp. 324, 501). Leduc did not consider a causal explanation for these phenomena, calling into question the validity of the generally accepted cell theory of Remak and Virchow of the 1850s, according to which cells arise only through the division of an existing cell (Deichmann, 2012).

Thompson's primacy of physics and form over chemistry and matter was strongly influenced by his leaning toward Platonic philosophy, in which forms (or ideas) are abstract entities that exist independently of the sensible world, and in which ordinary objects, imperfect and changeable, only faintly copy the perfect and immutable forms. Consequently, he regarded his task to be finished if he was able "to show that a certain mathematical aspect of morphology, to which as yet the morphologist gives little heed, is interwoven with his problems, complementary to his descriptive task, and helpful, nay essential, to his proper study and comprehension of Growth and Form" (Thompson, [1917] 1942, p. 1096).

The book is pervaded by poetic passages, for example, in his expression of admiration of the mysticism of numbers of the Pythagorean school. To him, "the concept of number as in the dreams and visions of Plato and Pythagoras," received the highest priority in the expression of knowledge (Thompson, [1917] 1942, p. 2), and he not only considered "number, order, and position" important for the exact knowledge of material things but also "square and circle" as decisive means to understand the universe, because "the harmony of the world is manifest in Form and Number, and the heart and soul and all the poetry of Natural Philosophy are embodied in the concept of mathematical beauty" (Thompson, [1917] 1942, pp. 10961097). According to Abzhanov (2017, p. 4286), for Thompson the study of natural laws and patterns could greatly benefit from the esthetic awareness and appreciation of nature. 
The assessment of the scientific impact of "On Growth and Form" poses a dilemma. On the one hand, the book has been widely admired and praised by a number of renowned scientists. On the other, "Growth and Form" had little direct scientific impact on research and never contributed to mainstream experimental biology at any time. However, there is a recent renewed appreciation for the mathematical and physical approaches of Thompson and his predecessors such as Wilhelm His: Morphologists have begun to combine the old, largely metaphoric approach of Thompson and others with insights from molecular biology such as gene regulation and signaling molecules (Savin et al., 2011). According to Ball (2017), it was probably Thompson's indebtedness to the biomechanics of the early anatomists His and Wilhelm Roux that "ties Thompson most securely to the present," where the mechanics of cell structure has become a focus of cell biologists. Sepkoski (2012) considers Thompson's study, "despite its logical flaws," to have been "an important basis for the developing field of theoretical biology". In 1962, paleontologist David M. Raup was the first to make computational representations of sea shell shapes and used a logarithmic spiral to describe shells' growth (Raup, 1962).

The fascination with "Growth and Form" by scientists such as Peter Medawar, Stephen Jay Gould, and James Briscoe was, in part, due to Thompson's exquisite language and breadth of knowledge. It shows that appreciation of a scientific publication does not have to be due to novel methodologies or concepts or the correctness of the statements, but also to wisdom and style way of writing. According to Tanner (1988), “'D'Arcy was one of Peter's [Medawar] great heroes; not so much because of what he said perhaps, but for the beautiful prose of Growth and Form"-Medawar had described the book as "the finest work of literature in all the annals of science that have been recorded in the English tongue" (Briscoe and Kicheva, 2017). Certainly, Medawar, the strongest follower of Popperian philosophy among biologists, could not have endorsed Thompson's methodology, in which experiments and hypothesis testing did not play any role.

Zoologist Wallace Arthur praises Thompson's writings as "great literature as well as great science," but criticizes that Thompson made no attempt to provide causal explanations in either the original or later editions of his book (Arthur, 2006). In relation to his "theory of transformations," Thompson might have included embryology as an explanatory category. But Thompson's comparisons of form are confined to adult forms and do not include juvenile ones. Wallace considers Thompson's "theory of transformations" highly inspiring, although it did not contribute to "experimentation to reveal the causal nature of the transformations" (Arthur, 2006). According to Peter Medawar, Thompson's method of "transformation", was hardly used by others (Clark and Medawar, 1945, p. 157).

Briscoe and Kicheva (2017) explain the influence of the book, in part, with the "clarity of its illustrations and the elegance and accessibility of the writing," as well as the originality and breadth of the subject matter. For them, the main importance of Growth and Form was the demonstration of how mathematical reasoning could be used to provide succinct descriptions of living forms and to reveal elegant simple answers to seemingly complex problems. In their research, Briscoe and Kicheva (2017) combine mathematical modeling with experimentation to elucidate the mechanisms of neural tube development and their genomic causes: "Experiments, combined with mathematical analysis of the neural tube regulatory network, have provided an explanation for several seemingly counterintuitive aspects of neural tube development." ... "The mathematical model suggested that the dynamics of the transcriptional network ... in the ventral neural tube could account for both spatial and temporal patterns of gene expression."

The fact that this approach is fundamentally different from Thompson's phenomenological one, in which the search for mechanisms and their causes are entirely missing, does not diminish both Briscoe's and Kicheva's admiration for Thompson's book. The quotation from Growth and Form, with which they conclude their article on Thompson, shows that scientists can be inspired by ideas that transcend laboratory work: "For the harmony of the world is made manifest in Form and Number, and the heart and soul and all the poetry of Natural Philosophy are embodied in the concept of mathematical beauty" (Briscoe and Kicheva, 2017).

Apart from a few mathematicians (see section 2.2.2), Stephen J. Gould appears to be the only renowned scientist whose major concepts were inspired by Growth and Form:

(1) Thompson rejected Darwin's idea of evolution through natural selection. In analogy to the Platonic idea of pure form which implied that mathematical shapes cannot be transformed through gradations, and according to his "theory of transformations" in which he geometrically transformed adult forms into one another, Thompson concluded that major discontinuities in organic form cannot be bridged by the intermediates in Darwinian theory (Gould, 1971). He believed that major evolutionary 
changes often occur suddenly. In 1972, Gould and Eldredge published their hypothesis of punctuated equilibrium, according to which major morphological changes in evolution are rare and relatively sudden events (Eldredge and Gould, 1972). This hypothesis fundamentally opposed the neoDarwinian concept of phyletic gradualism that perceives evolutionary changes as gradual and continuous. Although they did not cite Thompson and, of course, based their hypothesis of punctuated equilibrium on evidence from paleontology, not Greek philosophy, Thompson might have been one of the sources of inspiration for Gould, who in an article a year earlier expressed appreciation of Thompson's criticism of gradualism (Gould, 1971).

(2) Thompson's (mistaken) emphasis on organisms' forms resulting from the direct impact of physical forces contradicted the Darwinian explanation of form resulting solely from ancestry and natural selection. Citing Thompson, Gould and Lewontin in 1979 published their criticism of the neoDarwinian adaptationist program in which they pointed out that organisms' body plans (Baupläne) are constrained by "phyletic heritage, pathways of development, and general architecture to such an extent that these constraints delimit pathways of evolutionary change and may become more important than the selective forces (which they, unlike Thompson, considered highly relevant as well) (Gould and Lewontin, 1979).

In biology, form and matter are inseparable; in the words of Morange (2008) "the living world is [...] the product, both structurally and functionally, of a particular chemistry." Thompson's focus on morphological phenomena, his lack of experimental testing of his claims, and his disregard for the importance of chemistry and the interrelationship of form and matter are among the reasons why, despite the popularity of his book, his ideas had little impact on the lasting advances of biology in the first decades of the 20th century, such as genetics, experimental embryology, and biochemistry. This was expressed in a review of the 1942 edition: "The ideas of On Growth and Form have played little part in the spectacular advances of biology since the book was first written" (Wilson, 1944).

Stephen J. Gould perceived vital similarities between Thompson's approach and Watson and Crick's generation of the double helix model in 1953, namely the emphasis on form and the marginalization of a detailed chemical analysis: According to Gould (1971), Watson's book The Double Helix (1968) in which he described his and Crick's research leading to the discovery of the model stands second only to Thompson's Growth and Form as a 20th century "paean of form," because it described the pursuit of a (molecular) "shape too pretty not to be true". Watson's success, according to Gould, "emerged directly from a concern with form, from a methodology that prescribed the construction of models in preference to the search for a more subtle chemistry" (Gould, 1971).

However, contrary to Gould's view, a closer view on Thompson's modeling of form and Watson and Crick's modeling of a molecular form reveals fundamental differences that also may explain the different impacts these two models had on biological research. The assumption that Watson and Crick successfully constructed their model without detailed knowledge of the biochemistry and physical chemistry of its constituents is a widespread myth that is also shared by Gould. It ignores the fact that the emergence of the beautiful molecular shape was preceded by arduous research for "a more subtle chemistry" in which Watson and Crick consulted numerous colleagues and textbooks of theoretical chemistry (Morange, 2008, p. 12). The comparison of Thompson's and Watson and Crick's modeling exemplifies with great clarity deep conceptual and epistemological differences: The DNA double helix model was not a mathematical description, as were Thompson's models, but an experimentally testable hypothesis of a molecular structure and function. It was not a simulation of an organic feature, but it was based on the inductive integration and "consilience" of experimental observations (biochemical, physicochemical, and X-ray crystallography) with chemical theory. It could, for example, not have been derived by the figures provided by X-ray crystallography alone (Brenner, 2010). Unlike Thompson's models the double helix model had strong predictive capacities: it predicted testable mechanisms of the functions of gene replication, mutation, and information storage.

Decades later, Thompson's approach was taken up by some mathematicians and theoretical biologists. Meinhardt and colleagues derived their method of modeling sea shells from Thompson's detailed description and measurement of shell geometry (e.g., Fowler et al., 1992). Meinhardt and other theoretical biologists also created models for the generation of biological patterns by graded morphogens based on the idea of self-organization. Like Thompson, they disregarded the causal role of genes in their developmental models. 
One of the mathematicians who were influenced by Thompson was Alan Turing, whose theoretical model of development will be briefly described next.

2.2.2. Alan Turing's computational modeling of development. Mathematician and computer scientist Alan Turing most likely began to work on morphogenesis, the generation of form in a developing organism, in the context of his work on brain development (Fox Keller, 2002, p. 89). In this work he introduced reaction-diffusion equations into the modeling of development. Turing's model showed mathematically that in a system of two or more diffusing reagents a pattern of high and low concentrations may spontaneously emerge from an initially uniform distribution (Turing, 1952). The idea behind this extremely simplified model of morphogenesis was that two homogeneously distributed substances within a certain space, one "locally activated" and the other capable of "long-range inhibition," can produce novel shapes and gradients. The results of these substance interactions are dependent on just four variables per substance - the rate of production, the rate of degradation, the rate of diffusion, and the strength of their activating/inhibiting interactions (see also Cable, 2010).

Turing did not consider the growth of the embryo and restricted himself to the chemical dynamics of a nongrowing embryo, assuming that the generation of inhomogeneous patterns of the substances shaped the physical structure of the embryo. He called these substances morphogens, "form producers," "simply the kind of substance concerned in this theory," for example, a hormone, skin pigment, or a gene. Since he assumed the role of genes to be purely catalytic, "they may be eliminated from the discussion" (Fox Keller, 2002, p. 93). Postulating the presence of two morphogens with different diffusion constants, the model was represented by two coupled differential equations. Turing suggested that the system of reaction and diffusion of morphogens might account, for example, for the generation of tentacle patterns on Hydra and for whorled leaves.

Because of the discrepancy between model and reality, the reaction-diffusion system (and other mathematical-biochemical models) was at first poorly received in the biology community [for a detailed historical analysis of Turing's model, see Fox Keller (2002)]. Turing's article was barely cited by biologists until 1972 but impacted the study of chemical systems. In 1972, Ilya Prigogine and his school of the irreversible thermodynamics of complex systems made the model popular for some time. Reaction-diffusion studies, in particular, on pattern formation in butterfly wings and animal coats increased. Biologists applied Turing's model mostly to study the patterns of body segmentation in Drosophila (Fox Keller, 2002, pp. 110-111). However, the basic questions of development could not be solved by Turing's methods for two reasons:

(1) The model disregarded the role of genes as causal factors for development. Strong evidence of chromosomes as causal factors of development had already been presented by Theodor Boveri and Edward Wilson at the beginning of the 20th century. The importance of genes for development, including pattern formation, was demonstrated from the 1960, among others, by Edward Lewis and later Christiane Nüsslein-Volhard. There were debates early on, for example, by Boveri, Wilson, and Hans Driesch, about the reasons why development usually resulted in a functioning organization of the same species like the parents. While Driesch finally resorted to vitalism, other biologists concluded that it was due to chromosomes as a preformed nuclear agency (Deichmann, 2014).

(2) The unfertilized egg, as scrutinized, for example, in the work by Nüsslein-Volhard, was not considered a homogeneous sphere but rather a highly organized structure containing, among other things, a spatial pattern of information carrying mRNA and proteins (Fox Keller, 2002, p. 111).

In contrast, Turing's model was a purely speculative model of the generation of form by selforganization out of nothing. It was not based on biological data, but could mimic some patterns. As with the purely theoretical models of the genetic code, the model was mathematically more elegant than nature as illustrated by the following case, summarized in Fox Keller (2002, p. 340), which also points to the limited validity of simulation models in biology: According to computer simulations of pathways of the evolution of pattern-forming networks by I. Salazar-Ciudad et al. in 2001, stripe patterns of more than three stripes require fewer genes and are easier to generate by reaction-diffusion systems than by the hierarchical gene networks that actually account for the patterns in Drosophila. Answering the question of why Drosophila does not use reaction-diffusion mechanisms to generate its segments, the authors suggested that in the course of evolution, reaction diffusion mechanisms were replaced by hierarchical networks, which were 
selected for robustness with respect to mutations and developmental noise (Salazar-Ciudad et al., 2001; Fox Keller, 2002, p. 340).

In the present, the opinions regarding the validity of Turing's model differ widely.

In Endless Forms Most Beautiful, Sean B. Carroll writes,

“...Many theoreticians sought to explain how periodic patterns could be organized across entire large structures. While the math and models are beautiful, none of this theory has been borne out by the discoveries of the last twenty years. The mathematicians never envisioned that modular genetic switches held the key to pattern formation, or that the periodic patterns we see are actually the composition of numerous individual elements" (Carroll, [2006] 2007, p. 123). In other words, mathematically elegant but biologically irrelevant.

Other scientists disagree with Carroll's conclusions. They are of the opinion that although fly embryogenesis cannot be explained by reaction-diffusion systems, other patterns can be, such as hair follicle distribution in mice or stripe formation in zebrafish. However, the use of reaction-diffusion models for pattern formation in a genetic system is limited. But, as Cable (2010) suggested, even if the model "does not apply to much of anything, it can still generate useful questions that biologists can further explore.

Turing's models are similar to D'Arcy Thompson's in that both simulate the formation of patterns and forms. They emphasize the relevance of mathematics for tackling biological questions. However, because they are not based on experimental data and hypotheses, and do not take into account the causal role of genes, they do not explain mechanisms and causes of development.

\section{TWENTY-FIRST CENTURY MODELS IN RESEARCH ON COMPLEX BIOLOGICAL OBJECTS}

\subsection{Eric Davidson's GRN model of development}

Starting in the 1960s, molecular biological research, which had previously focused on microorganisms and viruses, broadened its scope to include the search for molecular explanations of phenomena that only occur in higher organisms, for example, development. New techniques enabled system approaches, for example, through the study of the expression of a large number of genes at the same time, leading to research along very different methodological and philosophical lines.

In this section I scrutinize Eric Davidson's systems approach as one that pursued the same mechanistic goals as 20th century experimental biology. Davidson created the concept of developmental GRNs in the early 2000s. Basic knowledge of genetic regulation in the development of higher organisms had already been obtained from Drosophila, in particular by Christiane Nüsslein-Volhard and Eric Wieschaus; they also demonstrated the hierarchy of maternal genes in the embryo that ultimately played an important role in Davidson's GRN. Early models of the temporal dynamics of already known gene networks in development by Garett Odell and his coworkers in 2000, who were using continuous differential equations, included only few genes (Fox Keller, 2002, pp. 250-251). Davidson was the first to achieve an almost complete model of a regulatory gene network for the development of a particular phenotype (endomesoderm specification) and to construct a mathematical model to account for observation in a complex biological object. This was also due to the fact that, using big sequencing and expression data, he made a large quantitative step from few regulatory genes to networks of hundreds of genes.

A world leader in molecular embryology, Davidson demonstrated that, at least in sea urchins, early development is entirely regulated by the genome, to him a logical necessity and requirement for evolution, because without such a genomic regulatory program it cannot be ensured that within each species the outcome of development is extremely reproducible. The existence of a hierarchical GRN for development that Davidson together with his colleague Roy Britten already postulated in 1969 (Britten and Davidson, 1969) was widely confirmed by many molecular biologists and by the authors themselves in the following decades.

Early on, Davidson joined forces with physicists and computer scientists to integrate computer-generated big data into a systems approach that was based on experiments and aimed at elucidating mechanisms and causal relationships. In 2006 he introduced the term "regulatory genome" for the interactions between regulatory genes and their products during development (Davidson, 2006). This concept was conceived and 
developed through decade-long, painstaking experimental research by Davidson and his collaborators. They systematically examined the cell-type specific gene expression patterns before moving on from the "gene-by-gene characterization of the sea urchin embryo to full comprehensiveness" (Rothenberg, 2016). This systems approach was made possible when sequencing data of the whole sea urchin genome became available.

Observations and descriptions were crucial as a starting point. According to Davidson (2016), descriptive observations, often derided as intrinsically inferior to "problem solving," received a new meaning in a systems approach: "The whole interaction system cannot be studied effectively until the whole set of its components and the output (behavior) is correctly measured/observed." Following the acquisition of observational data, hypotheses were indispensable. A perturbation analysis was essential because "only by deliberate experimental perturbation and predictive challenge of the system can the mechanisms by which it operates be revealed" (Davidson, 2016).

To test the proposed network, and because of the scale of the regulatory systems that control development, mathematical modeling was required. Davidson's first models in the 1990s not only contained logic functions (AND, OR, NOT) between the input of different regulatory proteins (transcription factors) but also assumed that they were quantitatively modulated; thus he and his collaborators created systems of differential equations with continuously variable inputs and outputs [Bolouri and Davidson (2002); see the overview by Rothenberg (2016)].

However, Davidson and his colleagues were aware of the fact that the key rate constants and concentrations needed to render these models predictive did not exist. As a consequence, Davidson envisaged that a Boolean model, in which the status of each gene is assumed to be either "on" or "off," might be sufficient as a predictive systems model of development. This was a major conceptual change (Rothenberg, 2016). A rationale for the decision for a Boolean model was that, among other things, GRNs result in discrete patterns of gene expression "which in Boolean models can be captured as alternative states of genes" (Peter and Davidson, 2015, p. 309).

Together with Isabelle Peter, Davidson converted the whole GRN system into a Boolean model, a "grueling effort lasting many months of concentrated work" (Peter and Davidson, 2015). This model contained all current data for the logic of transcriptional inputs at each gene (cis-regulatory) system and also for the location of each cell at each time point of development. The model, published in 2012 (Peter et al., 2012), made it possible, for the first time, to test whether the (endomesoderm) gene model that Davidson had experimentally created and refined for 10 years was (almost) complete and predictive. The first results of the Boolean modeling showed that there were indeed only few inconsistencies between model predictions and measured in vivo gene expression. This meant not only that the key regulatory elements of the GRN and their interactions were almost complete but also that other factors such as changes in chromatin structure did not appear to be relevant at this early developmental stage.

Among the characteristics of this Boolean model are: (1) The model was based on a causal-mechanistic concept, that is, on experiments that proposed linkages based on perturbations, not correlations. It provided a means for experimentally testing the relevance and consistency of the GRN concept, thus fundamentally contrasting with models that merely simulated phenomenological features without analyzing their mechanisms and causes. (2) The model aimed at encompassing all relevant genes of the network and their interactions. The background for this aim was Davidson's vision of creating a model that was fully rooted in the genomic sequence, thus showing that early development was controlled by the genome. This aim contrasted with models that focus only on parts of the process, for example, on signaling events without asking why the signal is expressed in the sending cells or what it does to gene activation in the receiving cells. Aware of the fact that (genomic) causality and mechanism cannot be arrived at if only a minor fraction of the components active in a process are encompassed in the analysis (Davidson, 2016), the aim of completeness was of great importance.

Davidson's outlining of a mechanism of completeness that accounted for any possibility, or, as he put it, without leaving any room for the appearance of "black swans," was also philosophically motivated, namely by his wish to overcome Popper's criticism of induction in science (Davidson, 2014)-Popper rejected induction as a means of creating, as well as of testing, hypotheses. Davidson's strong emphasis on hypothesis (even bold hypotheses), predictability, and hypothesis testing had clear Popperian traits, but his emphasis on induction contradicted Popper. Davidson considered late twentieth century experimental analyses of developmental biology to have lived in the shadow of Karl Popper's thesis that "a single instance to the contrary can prove an inductive mechanistic idea wrong," or, as Popper put it, that one black 
swan can falsify the inductive assumption that "all swans are white" (Davidson, 2014). Such an epistemology, Davidson argued, might have some value in pregenomic research that "focused on a given gene or a given small set of genes" where it could not be predicted "where the next Australian black swan would turn up." But the advent of the possibility that all parts of the developmental system can be included in mechanistic analysis, in principle, offered a probable counter to the concern that "black swans" might falsify the hypothesis (Davidson, 2014).

Clearly, Davidson's and other biologists' reliance on large amounts of experimental data as a basis for generating hypotheses and models-in Davidson's words (2016), "In basic science, hypothesis formation is an inferential, inductive, creatively novel proposition of mechanism"-contradicts Popper's dictum that the basis for the advance of science is not the accumulation of more and more perceptual experiences.

\subsection{GRN models differ between systems: The example of Ellen Rothenberg's GRN model of the hematopoietic system}

The fruitfulness of the concept of developmental GRNs has been widely acknowledged by many biological scientists, as well as by historians of biology (e.g., Fox Keller, 2002; Cameron, 2015; Rothenberg, 2016; Buckingham, 2017). It has been taken up and modified by many biologists, for example, by James Briscoe in his research on the development of the central nervous system (Gouti et al., 2017) and by Ellen Rothenberg in her research on hematopoietic stem cell differentiation (Rothenberg, 2017a). In much of the research on developmental GRNs, mathematical modeling has become crucial. It is interesting to note that while the underlying research in general is mechanistic, that is, aims at finding mechanisms for how the hierarchical genomic networks through the interaction of the relevant regulatory genes give rise to the development of particular features of the embryo, the characteristics of the networks sometimes differ substantially depending on the function for which they have been evolved.

Ellen Rothenberg, who has been working on the development of the mammalian postnatal hematopoietic system and has not yet completed a fully elaborated model of her system, reflected on models' differences and on an evolutionary explanation for the explanatory powers of different GRN models: She agreed with Eric Davidson that "there is little doubt that ultimately there are gene networks that drive development." But she also draws attention to the fact that the analytical and predictive models that capture essential features of GRNs need to be considered separately for different modes of development. This is because "the importance of quantitative effects on target gene expression as opposed to qualitative ones, the precision of timing of state changes, and the nature of negative regulation can all differ considerably between biological systems" (Rothenberg, 2017a). Thus, while "the sea urchin embryo is highly precise and accurate about both cell fate determination and timing of differentiation of every cell type in the embryo, and has evolved to accomplish this by relative insensitivity to absolute levels of expression of key regulators," the mammalian postnatal hematopoietic system "has evolved to emphasize plasticity and environmental responsiveness at the expense of precision in both timing and cell fate determinism" (Rothenberg, 2017a).

This means that known components of certain developmental pathways through known mechanisms could put cells into states from which the future is not intrinsically determined. Thus their responses to environmental influences in the organism decide their developmental outcome. According to Rothenberg, this "developmental stochasticity" is not a placeholder for all the quantities that cannot be measured but "a specific, evolutionarily selected outcome of a regulatory state" (Ellen Rothenberg to the author, June 17, 2017).

Rothenberg holds that network models cannot be complete, that is, include all genomic genes because most genes are not relevant to a given developmental choice or progression step. She emphasizes that some of the art of network analysis is to use sufficient prior knowledge to focus on genes that are most likely to be functionally important. She holds that network models, even if they are incomplete, are useful because they are crucial to capturing large bodies of complex experimental results in a comprehensive way; they can clarify the understanding of biological relationships that do not fit a preconceived notion; they can point the way to experiments that improve understanding; their comparison with observed gene expression patterns can reveal what kinds of functions are missing (Rothenberg, 2017).

\subsection{Comparison of models of complexity with earlier models}

In their being based on experimental data, containing causal propositions and predictions that can be experimentally tested and providing a conceptual framework for further experimenting, models in causal- 
mechanistic systems research on developmental GRNs show many similarities to the successful 20th century models.

Major differences derive from the fact that the GRN models-like all models of complex biological objects-are based on much larger amounts of complex experimental data than earlier mathematical models. Data accumulation and induction play a larger role than in the past. Still, the models are simplifications as well. This is despite the aim of completeness regarding key regulatory elements in Davidson's network models: In these models, too, only a limited number of genes was included in the perturbation based networks; they were selected for territory specificity, excluding even genes that encoded transcription regulators that were uniformly expressed through the embryo; likewise, quantitative changes of transcription factors were not included (Rothenberg, 2016). According to Jeremy Gunawardena, Davidson's models conceal many assumptions, most importantly that they are digital abstractions: One of the central assumptions of his Boolean GRN model is that genes are on or off, but in reality, this distinction is not always so clear. Thus, despite their high level of complexity, Davidson's models contain simplifications (Jeremy Gunawardena to the author, July 9,2018). This does not affect the usefulness of the models for handling large amounts of sequencing and experimental data, their fruitfulness for predictions, and "experimentally testing the relevance and consistency of [a causal-mechanistic] concept" (Peter and Davidson, 2015).

Although they are based to a much larger extent on description and inductive reasoning than earlier models, the GRN models are not mathematical descriptions, but predictive hypotheses of reality. They fundamentally contrast with claims by some engineers and computational biologists that mathematical descriptions have started to replace causal-mechanistic explanations in biology. According to the engineers and computational biologists Edward R. Dougherty and Michael L. Bittner, biology is in need of scientific theories "to be expressed mathematically without appeal to commonsense categories of understanding, such as causality" (Dougherty and Bittner, 2010). They confine biological knowledge to the mathematics involved in "modeling the relations that characterize regulatory knowledge": "Scientific knowledge [in biology] is constituted by the mathematics of stochastic dynamical systems, which model the overall relational structure of the cell and how these structures evolve over time, stochasticity being a consequence of the need to ignore a large number of factors" (Dougherty and Bittner, 2010).

The extent to which such an engineering approach may be successful for the modeling of certain cellular features cannot be assessed here. It is true that there is a "commonsense" category of causality, and as Judea Pearl and Dana Mackenzie pointed out, this "causal wiring produces systematic probabilistic mistakes" (Pearl and Mackenzie, 2018, p. 183). But, as these authors also emphasize, causal models are crucially important as a prerequisite for interpreting data, thus "hypothesizing on how things operate in the real world" (Pearl and Mackenzie, 2018, p. 316).

Dougherty and Bittner's exclusion of causality-and thus of processes of intervention such as perturbation experiments - as well as their marginalization of biological knowledge, render a causal mechanistic understanding of phenomena impossible. An example is the disregard for evolution: As mentioned before, the stochasticity in certain developmental models, such as in Ellen Rothenberg's model of the hematopoietic system, can be the result of evolutionary selection, not a placeholder for unmeasurable quantities.

\section{MARGINALIZATION OF MODELING}

Unlike in the models discussed earlier, and in other biological research that combines the use of big data technology with hypothesis formation and experimental testing, the availability of large amounts of sequencing and expression data has been used to proclaim a new era of science based on correlations only.

Discovery science, "a scientific methodology, which emphasizes analysis of large volumes of experimental data with the goal of finding new patterns or correlations," Discovery Science (2016) is an example. Prior knowledge is no longer required; data mining proceeds with the help of "specialized machine learning algorithms for automated hypothesis forming and automated theorem proving." Mathematical modeling becomes obsolete.

The creation of a new belief system, such as the belief in algorithms without subsequent critical experimental testing of the hypotheses generated by the computer, deviates fundamentally from conventional research and the philosophical premises that guided it. The notion of science in which observational data themselves can lead to discovery has been labelled as a new era of empiricism: the "volume of data accompanied by techniques that can reveal the truth enables data to speak for themselves free from theory" (Kitchin, 2014). 
The conviction that data alone can provide the right answers to scientific questions is not an invention of 21 st century "big data" science but can be traced back to the founding fathers of statistics in the early 20th century, such as Karl Pearson and Ronald Fisher, who "purged [science] of the language of cause and effect" (Pearl and Mackenzie, 2018, p. 127). The notion of scientific truth as empirical truth, not related to unchangeable scholastic first principles such as that of "substantial form," was propagated in the 17th century by Francis Bacon (1561-1626), whose epistemology strongly influenced subsequent empirical sciences. However, the idea of observations leading directly to discovery disregards the fact that for Bacon, too, data are not to be obtained arbitrarily, "groping, as of men in the dark," but guided by reason and accompanied by experiments. According to Hacking ([1983] 2007, p. 247)., Bacon "does not fit well into the simple dichotomies of inductivism and deductivism"; he resented purely speculative theories, as well as pure empiricism that relied on sense data only, and even "knew the value of learning by refutation."

Description and induction, in addition to hypothesis testing, have continuously played an important role in experimental biology, later in molecular biology and molecular system biology. In marked contrast to what is today called big data empiricism, the inductive method has always been guided by researchers' ideas and reason, and the results of inductive reasoning were experimentally tested (except for purely statistical research). Although in many cases novelties were discovered unexpectedly, knowledge was required for understanding, as is expressed in Pasteur's famous dictum that in the fields of observation chance favors only the prepared mind.

In the widely-cited article "The End of Theory," journalist and former editor of "Wired" magazine Chris Anderson proclaimed that big data should govern the science of the future and model generating should be abandoned altogether (Anderson, 2008). According to him, "there is now a better way." Petabytes allow us to say: "Correlation is enough. We can stop looking for models. We can analyze the data without hypotheses about what it might show. We can throw the numbers into the biggest computing clusters the world has ever seen and let statistical algorithms find patterns where science cannot."

Developments in computer science and engineering have indeed pushed pattern recognition in many fields of science far beyond the ability of humans. However, a complex science such as biology is not confined to pattern recognition. Knowledge of mechanisms is crucial to answering questions about issues such as the causal role of genes and genetic variation in development or the effects of perturbations or diseases on a system. Eric Davidson's success in creating a GRN model that predicts responses of a developmental system to perturbations is a case in point. His work and that of other experimental system researchers demonstrate the relevance of Popper's principles of hypothesis creation and experimental testing not only in 'reductionist' biological research but also in that part of systems research that is not merely descriptive but aims at finding causal mechanistic explanations.

Their works also highlight the requirements that Michael Polanyi deemed essential for successful research, namely knowledge, competence, and commitment to scientific beliefs, that is, a passionate commitment to "a vision of reality" (Polanyi, [1959] 1962, p. 64). Interestingly, computer scientist Judea Pearl likewise emphasizes that unlike in correlation, "subjective commitment" is required for causal analysis: "Where causation is concerned, a grain of wise subjectivity tells us more about the real world than any amount of objectivity" in the model-free methods. However, he adds that once people agree on their assumptions, causal inference "provides a hundred per cent objective way of interpreting new evidence" (Pearl and Mackenzie, 2018, pp. 89-90).

Moreover, confining biology to pattern recognition and correlations would mean disregarding basic biological principles, among them the hierarchy in biological systems and, as shown previously, their evolutionary origin. This has also resulted in their noisiness (e.g., transcription factors that can interact with many nonfunctional sites) and the different meanings of the term "function" (Morange, 2014). To allow "data to speak for themselves, free from theory" means not only giving up science as an intellectual enterprise, aimed at finding explanations, but also calling into question the notion of scientific truth that can be subjected to scrutiny.

\section{FINAL REMARKS}

While the number of articles using mathematical modeling is decreasing in contrast to those using data and correlations, this article has shown that in the era of big data, too, modeling is still at the heart of basic science that aims at providing causal-mechanistic explanations. The statistician George Box, in an often 
cited phrase, expressed the opinion that good models cannot represent the truth of completeness of a phenomenon: "All models are wrong but some are useful: Now it would be very remarkable if any system existing in the real world could be exactly represented by any simple model. However, cunningly chosen parsimonious models often do provide remarkably useful approximations. [...] For such a model there is no need to ask the question 'Is the model true?' If 'truth' is to be the 'whole truth' the answer must be 'No.' The only question of interest is 'Is the model illuminating and useful?", (Box, 1979). This question is also relevant for biological models of complex systems.

It is important to remember that knowledge is not composed only of data. In the opinion of Judea Pearl, it is causal explanations that "make the bulk of our knowledge," and "data alone cannot make up for lack of scientific knowledge" (Pearl and Mackenzie, 2018, pp. 21, 28). Brenner (2010) best summed up the importance of theory (this implies causal models) for 21st century biology: "We should welcome with open arms everything that modern technology has to offer us but we must learn to use it in new ways. Biology urgently needs a theoretical basis to unify it and it is only theory that will allow us to convert data to knowledge."

\section{AUTHOR DISCLOSURE STATEMENT}

The author declares there are no competing financial interests.

\section{REFERENCES}

Abzhanov, A. 2017. The old and new laws and faces of morphology: The legacy of D'Arcy Thompson's 'theory of transformations' and 'laws of growth.' Development 144, 4284-4297.

Anderson, C. 2008. The end of theory: The data deluge makes the scientific method obsolete. June 23, 2008. http:// www.wired.com/2008/06/pb-theory/ Last accessed on Nov. 22, 2016.

Arthur, W. 2006. D’Arcy Thompson and the theory of transformations. Nat. Genet. 7, 401-406.

Ball, P. 2017. In retrospect-On growth and form. Nature 494, 32-33.

Bolouri, H., and Davidson, E. 2002. Modeling transcriptional regulatory networks. Bioassays 24, 1118-1129.

Box, G.E.P. 1979. Robustness in the strategy of scientific model building, 201-236. In Launer, R.L., and Wilkinson, G.N., eds. Robustness in Statistics. Academic Press, San Diego.

Brenner, S. 1999. Theoretical biology in the third millennium. Philos. Trans. R. Soc. Lond. B 354, 1963-1965.

Brenner, S. 2010. Sequences and consequences. Philos. Trans. R. Soc. B 365, 207-212.

Briscoe, J., and Kicheva, A. 2017. The physics of development 100 years after D'Arcy Thompson's “On Growth and Form". Mech. Dev. 145, 26-31.

Britten, R.J., and Davidson, E.H. 1969. Gene regulation for higher cells: A theory. Science 165, 349-357.

Buckingham, M. 2017. Gene regulatory networks and cell lineages that underlie the formation of skeletal muscle. Proc. Natl. Acad. Sci. U. S. A. 114, 5830-5837.

Cable, K.W. 2010. Alan Turing's Reaction-Diffusion Model-Simplification of the Complex. Available at: www.phylogenous.wordpress.com/2010/12/01/alan-turings-reaction-diffusion-model-simplification-of-the-complex. Accessed January 9, 2019.

Cameron, A. 2015. Eric H. Davidson (1937-2015). Systems biologist who described gene regulatory networks. Nature 526, 196.

Carroll, S.B. [2006] 2007. Endless Forms Most Beautiful. Phoenix, London.

Crick, F. 1958. On protein synthesis. Symp. Soc. Exp. Biol. xii, 138-163.

Davidson, E.H. 2006. The Regulatory Genome: Gene Regulatory Networks in Development and Evolution. Academic Press, San Diego, CA.

Davidson, E. 2014. Brief notes on the meaning of a genomic control system for animal embryogenesis. Perspect. Biol. Med. 57, 78-86.

Davidson, E.H. 2016. Genomics, "Discovery Science," systems biology, and causal explanation. What really works? Perspect. Biol. Med. 58, 165-181.

Deichmann, U. 2010. Gemmules and elements: On Darwin's and Mendel's concepts and methods in heredity. J Gen. Philos. 41, 31-58.

Deichmann, U. 2012. Crystals, colloids or molecules? Early controversies about the origin of life and synthetic life. Perspect. Biol. Med. 55, 521-542. 
Deichmann. U. 2014. The Concept of the causal role of chromosomes and genes in heredity and development: Opponents from Darwin to Lysenko. Perspect. Biol. Med. 57, 57-77.

Deichmann, U. 2017. Hierarchy, determinism, and specificity in theories of development and evolution. Hist. Philos. Life Sci. 39, 33.

Discovery Science. 2016. Available at: www.en.wikipedia.org/wiki/Discovery_science. Accessed January 9, 2019.

Dougherty, E.R., and Bittner, M.L. 2010. Causality, randomness, intelligibility, and the epistemology of the cell. Curr. Genomics 11, 221-237.

Dougherty, E.R., and Bittner, M.L. 2011. Epistemology of the Cell: A Systems Perspective on Biological Knowledge. IEEE Press Series in Biomedical Engineering, Wiley, Piscataway, NJ.

Droescher, A. 2015. Gregor Mendel, Franz Unger, Carl Nägeli and the magic of numbers. Hist. Sci. 53, $492-508$.

Eldredge, N., and Gould, S.J. 1972. Punctuated equilibria: An alternative to phyletic gradualism, 82-115. In Schopf, T.J.M., ed. Models in Paleobiology. Freeman Cooper, San Francisco, CA.

Fowler, D.R., Meinhardt, H., and Prusinkiewicz, P. 1992. Modeling seashells. ACM SIGGRAPH Comput. Graph. 26, 379-387.

Fox Keller, E. 2002. Making Sense of Life: Explaining Biological Development with Models, Metaphors and Machines. Harvard University Press, Cambridge, MA.

Gamow, G. 1954. Possible relation between deoxyribonucleic acid and protein structures. Nature 173, 318.

Gliboff, S. 1999. Gregor Mendel and the laws of evolution. Hist. Sci.37, 217-235.

Gould, S.J. 1971. D'Arcy Thompson and the science of form. New Lit. Hist. 2, 229-258.

Gould, S.J., and Lewontin, R. 1979. The spandrels of San Marco and the Panglossian paradigm: A critique of the adaptationist programme. Proc. R. Soc. Lond. B 205, 581-598

Gouti, M., Delile, J., Stamataki, D., et al. 2017. A gene regulatory network balances neural and mesoderm specification during vertebrate trunk development. Dev. Cell 41, 243-261.

Gunawardena, J. 2012. Some lessons about models from Michaelis and Menten. Mol. Biol. Cell 23, 517-519.

Gunawardena, J. 2013. Biology is more theoretical than physics. Mol. Biol. Cell 24, 1827-1829.

Gunawardena, J. 2014. Models in biology: 'Accurate descriptions of our pathetic thinking.' BMC Biol. 12, 29.

Johannsen, W. 1909. Elemente der exakten Erblichkeitslehre. Gustav Fischer. Jena.

Hacking, I. [1983] 2007. Representing and Intervening. Cambridge University Press, Cambridge, MA

Hayes, B. 1998. Computing science: The invention of the genetic code. Am. Sci. 86, 8-14.

Kay, L.E. 2000. Who Wrote the Book of Life? A History of the Genetic Code. Stanford University Press, Redwood City, CA.

Kitchin, R. 2014. Big Data, epistemologies and paradigm shifts. Big Data Soc. 1, 1-12.

Lewis, E.B. 1995. Nobel lecture. Available at: www.nobelprize.org/nobel_prizes/medicine/laureates/1995/lewislecture.html. Accessed January 9, 2019.

Medawar, P.B. 1945. Size, shape, and age. In Le Gros Clark, W.E., and Medawar, P.B., eds. Essays on Growth and Form Presented to D'Arcy Wentworth Thompson. Oxford University Press, Oxford. pp. 157-187.

Mendel, G. 1866. Versuche über Pflanzen-Hybriden. Verhandlungen des naturforschenden Vereins in Brünn, 4, 3-47, mendelweb.org. In English translation: Experiments in plant hybridization (read at the meetings of February 8, and March 8, 1865), mendelweb.org.

Mendel, G. 1866-1873. Letters to Naegeli. 1950. [Translated by Piterniak, L.K., and Piterniak, G.]. Genetics 35 (5, pt.2), 1-29.

Mirsky, A.E., and Pauling, L. 1936. On the problem of native, denatured, and coagulated proteins. Proc Natl. Acad. Sci. U. S. A. 22, 439-447.

Morange, M. 1998. A History of Molecular Biology. Harvard University Press, Cambridge, MA.

Morange, M. 2001. The Misunderstood Gene. Harvard University Press, Cambridge, MA.

Morange, M. 2008. À auoi sert l'Histoire des Sciences? Quae, Versailles.

Morange, M. 2014. Genome as a multipurpose structure built by evolution. Perspect. Biol. Med. 57, 162-171.

Pauling, L. 1940. A theory of the structure and process of formation of antibodies. J. Am. Chem. Soc. 62, $2643-2657$.

Pauling, L., and Campbell, D.H. 1942a. The production of antibodies in vitro. Science 95, 440-441.

Pauling, L., and Campbell, D.H. 1942b. The manufacture of antibodies in vitro. J. Exp. Med. 76, 211-220.

Pearl, J., and Mackenzie, D. 2018. The Book of Why. The New Science of Cause and Effect. Basic Books. New York, NY.

Peter, I., and Davidson, E.H. 2015. Genomic Control Process: Development and Evolution. Academic Press, Amsterdam, Netherlands.

Peter, I.S., Faure, E., and Davidson, E.H. 2012. Predictive computation of genomic logic processing functions in embryonic development. Proc. Natl. Acad. Sci. U. S. A. 109, 16434-16442.

Polanyi, M. [1958] 1962. Personal Knowledge-Towards a Post-Critical Philosophy. University of Chicago Press, Chicago, IL.

Raup, D.M. 1962. Computer as aid in describing form in gastropod shells. Science 138, 150-152. 
Rothenberg, E. 2016. Eric Davidson: Steps to a gene regulatory network for development. Dev. Biol. 412, S7-S19. Rothenberg, E. 2017. Fitting structure to function in gene regulatory networks. Hist. Philos. Life Sci. 39, 37.

Salazar-Ciudad, I., Solé, R.V., and Newman, S.A. 2001. Phenotypical and dynamical transition in model genetic networks. II. Application to the evolution of segmentation mechanisms. Evol. Dev. 3, 95-103.

Savin, T., Kurpios, N.A., Shyer, A.E., et al. 2011. On the growth and form of the gut. Nature 476, 57-62.

Schrödinger, E. 1944. What Is Life? The Physical Aspect of the Living Cell. Cambridge University Press, Cambridge, UK.

Sepkoski, D. 2012. Rereading the Fossil Record: The Growth of Paleobiology as an Evolutionary Discipline. University of Chicago Press, Chicago.

Tanner, J. 1988. Obituary-Sir Peter Medawar, 1915-1987. Ann. Hum. Biol. 15, 89.

Teicher, A. 2014. Mendel's use of mathematical modelling: Ratios, predictions and the appeal to tradition. Hist. Philos. Life Sci. 36, 187-208.

Thompson, D. [1917] 1942. On Growth and Form, 2nd ed. Cambridge University Press, Cambridge.

Turing, A.M. 1952. The chemical basis of morphogenesis. Philos. Trans. R. Soc. Lond. B Biol. Sci. B 273, 37-72.

Watson, J.D. 1968. The Double Helix. Weidenfeld and Nicolson, London.

Watson, J., and Crick, F. 1953. Genetical implications of the structure of deoxyribonucleic acid. Nature 171, 964-967.

Wilson, J.W. 1944. Review of the 1942 edition of D'Arcy Thompson, "On Growth and Form." Bull. Am. Math. Soc. $50,163-168$.

Address correspondence to:

Prof. Ute Deichmann

Jacques Loeb Centre for the History and Philosophy of the Life Sciences Ben-Gurion University of the Negev

Beersheba 8410501

Israel

E-mail: uted@post.bgu.ac.il 